\title{
Alcohol y drogas en las empresas de la Unión Europea
}

\author{
Cabrero, e. ${ }^{(1)}$, LunA, A. ${ }^{(2)}$ \\ (1) Doctor en Medicina. Médico del Trabajo. Jefe del Servicio Médico de Empresa de R.E.N.F.E. de la Comunidad Valenciana y Región de Murcia. \\ (2) Catedrático de Medicina Legal y Toxicología. Médico del Trabajo

\section{RESUMEN:}

La empresa es considerada por la Unión Europea como una de las instituciones más importantes para afrontar los problemas derivados del consumo de alcohol.

Las Leyes y normativas de los diferentes Estados miembros están adaptadas a las peculiaridades propias de cada uno de ellos, pero carecen de leyes específicas frente a este problema, basándose en leyes generales penales, de salud y seguridad y de empleo. Igualmente, poseen unas obligaciones limitadas en relación al tratamiento, la educación y el asesoramiento de los trabajadores, evidenciándose también posturas de castigo que afortunadamente son cada vez menos usadas. Sin embargo, las disposiciones que protegen la intimidad dificultan el control de los problemas relativos al consumo de alcohol y drogas que afectan a los trabajadores, si bien todos los Estados miembros parecen estar de acuerdo en la necesidad de tratar dichos problemas en los sectores de alto riesgo y de responsabilidad.

Los sindicatos y la mayoría de las empresas tampoco poseen políticas que afronten este problema, con excepción de las grandes empresas donde los sindicatos también tienen una mayor implantación.

Las Administraciones de los Estados miembros y las empresas y los sindicatos realizan actividades preventivas, en general, y de identificación de los trabajadores de riesgo, en particular, para que aquellos trabajadores con problemas derivados del consumo de alcohol y drogas sean tratados, recuperados y reinsertados social y laboralmente.

Palabras clave: prevención laboral, alcohol, drogas ilegales, Unión Europea, políticas preventivas

\section{ABSTRACT:}

The European Union considers the company as one of the most important institutions for confronting the problems derived from alcohol consumption.

Legislation and regulations in the various Member States are adapted to the peculiarities of each individual state but they all lack the specific legislation to deal with this problem as they are based on general penal, health and safety, and labour laws. Similarly, obligations are limited in respect of the treatment, education and assessment of employees, and also include penalising provisions that are, happily, being less and less applied.

Nevertheless, the provisions that protect the privacy of the individual lead to difficulties in controlling alcohol and drug associated problems that affect the labour force, even though all Member States appear to be in agreement on the necessity of treating these problems in sectors of high risk and responsibility.

In addition, the labour unions and the majority of companies do not have policies to deal with this problem, with the exception of the very large companies where the unions also have a greater influence.

The governments, companies and labour unions in the Member States carry out general preventive actions and identification of workers at risk in particular so that those with problems arising from alcohol consumption and drug use are treated, rehabilitated and socially and reinserted in the workforce.

Key words: workforce prevention, alcohol, illegal drugs, European Union, preventive policies
$\mathbf{L}$ a Unión Europea reconoce que una de las instituciones mas importantes para afrontar de forma integral los problemas derivados del abuso de sustancias son las empresas, y el conjunto del mundo laboral. Nuestra sociedad ha sido capaz de desarrollar estrategias de respuestas más elaboradas en el entorno escolar, o en otras instituciones sociales, que en la empresa. Así, la adquisición del conocimiento sobre las drogodependencias y las habilidades para afrontarlas que se han desarrollado en nuestro medio social, han ido muy por delante de las soluciones aplicadas en el mundo laboral.

Mientras que las repuestas sociales ante el problema del consumo abusivo de alcohol y drogas comenzaron a estructurarse en los años 60, las empresas pioneras en salud laboral, están llevando a cabo pro- 
gramas que lo afronten desde mediados de los 80 (1). La inmensa mayoría están aún sin ni siquiera recoger este punto en su normativa laboral.

La política de los Estados miembros de la Unión Europea sobre las situacio-nes que pueden afectar al trabajador derivadas del consumo de alcohol y drogas en el entorno laboral varía ampliamente, fundamentalmente en rela-ción con la legislación vigente y con el grado de implicación de las empresas en las políticas preventivas. Es diferente en cada Estado miembro y, aún den-tro de un mismo Estado, en las diferentes empresas del mismo, e incluso, nos atreveríamos a decir, que dentro de cada centro de trabajo.

Desde el punto de vista de los Estados miembros, hay algunos sin legislación específica al respecto (Grecia, Luxemburgo, Portugal, Reino Unido, República de Irlanda), aunque las grandes empresas adoptan normas internas sobre el tratamiento, la formación, el asesoramiento y la prevención del consumo de alcohol y drogas en lugar de trabajo. Otros países, aplican normas relativas a la conducción de vehículos bajo los efectos del alcohol (Bélgica, Países Ba-jos) o de los seguros (Alemania, Bélgica). Mientras que otros países sí poseen normas específicas dentro del Derecho Laboral (España o Francia), o desa-rrolladas o incluidas dentro de los Convenios colectivos (España o Italia) (2).

La Directiva marco 89/391/CEE y, en España, la Ley 31/1995 de Prevención de Riesgos Laborales, que es consecuencia del desarrollo de aquella, establecen que el empresario deberá garantizar la seguridad y la salud de los trabajadores y adoptará las medidas necesarias para la protección de la seguridad y de la salud, incluidas las actividades de prevención de los riesgos profesionales, de evitar los riesgos, planificar la prevención buscando un conjunto coherente, dar las debidas instrucciones a los trabajadores y, cuando confíe tareas a un trabajador, tomará en consideración las capacidades profesionales de dicho trabajador en materia de seguridad y salud (3).

En el momento actual la conceptualización del problema del trabajador que consume alcohol y/o, drogas, no se sitúa en el terreno represivo, afortunadamente nadie cuestiona la rentabilidad de un programa dirigido a la detección, tratamiento y reinserción de los traba-jadores afectados, no sólo para promo-cionar su salud, sino tam-bién para evitar consecuencias perjudiciales a él, a sus propios compañeros y/o a terceros (4-14). Pero además estos riesgos ad-quieren especial relevancia en aquellos trabajadores que manipulan sustancias potencialmente peligrosas (tóxicos, explosivos), alta tensión u otras energías peligrosas (presión, temperatura, radiación, etc.) y otros trabajos re-lacionados con la seguridad pública (grúas, obras públicas, control de tráfico aéreo, transporte público, policía, bomberos, militares, urgencias médicas), que no pueden permitirse estar bajo la influencia de las sustancias psicoacti-vas o de problemas derivados de su uso (abstinencia) cuando están de servicio (15-16).

Una minoría de los Estados miembros cuenta con leyes para prohibir la intro-ducción de bebidas alcohólicas en el lugar de trabajo (Bélgica, Francia y España), otra cosa es que se respeten de forma estricta. Pero, sólo Francia y España prohiben específicamente estar en estado de embriaguez en el lugar de trabajo $(2,17)$.

Algunas leyes de los Estados miembros imponen obligaciones específicas a los empresarios para que ofrezcan un lugar de trabajo seguro y saludable y a los trabajadores para que no dañen a otros. Es evidente que existe un riesgo objetivo en el caso de una intoxicación por drogas o por un consumo de bebidas alcohólicas $(2,3)$.

En España, la respuesta dada a las personas que presentan un patrón de consumo abusivo de substancias ha sido el reflejo del conocimiento y de la percepción que la sociedad, la empresa y la propia medicina la-boral ha ido adquiriendo.

El Estatuto de los Trabajadores (17), Ley 8/1980, recoge expresamente en su artículo 54, apartado 2 f, sobre despido disciplinario o lo que es lo mismo despido laboral procedente, esta causa de despido. Así, el aparato 1 expresa que "El contrato de trabajo podrá extinguirse por decisión del empresario, mediante despido basado en un incumplimiento grave y culpable del trabajador" y el aparato 2 "Se considerarán incumplimientos contractuales: a) Las faltas repetidas e injustificadas de asistencia o puntualidad al trabajo. b) La indisciplina o desobediencia en el trabajo. c) Las ofensas verbales o físicas al empresario o a las personas que trabajan en la empresa o a los familiares que conviven con ellos. d) La transgresión de la buena fe contractual, así como el abuso de confianza en el desempeño del trabajo. e) La disminución continuada y voluntaria en el rendimiento de trabajo normal o pactado. f) La embriaguez habitual o toxicomanía si repercuten negativamente en el trabajo".

La interpretación del Estatuto de los trabajadores, en los primeros de los primeros años 80, llevaba a que cualquier trabajador con problemas derivados del consumo de alcohol y drogas fuese despedido, no admitido o, en el mejor de los casos, se le ofreciese un cese pactado. Pronto, algunas empresas con posibilidades de acoplamiento de estos trabajadores, calificaron a estos trabajadores como no aptos, dado que su enfer- 
medad no les permitía el desempeño de sus funciones. Pero esta circunstancia sólo está al alcance de empresas de gran tamaño. Finalmente, tenemos que irnos a principios de los años 90 para encontrarnos, en nuestro país, con empresas que hubieran desarrollado un Programa contra las drogodependencias y el alcoholismo (18).

Hemos de recordar, que hasta este año, no se han hecho declaraciones a favor de derogar el punto f) del artículo 54.2 del Estatuto de los Trabajadores. Aunque tampoco se nos escapa que el trabajador enfermo por problemas de consumo de alcohol y drogas puede transgredir cualesquiera de los otros puntos de este artículo.

Estos programas preventivos y asistenciales pretenden la máxima recuperación y la reinserción laboral $y$, por ende, social del trabajador que ha estado afectado en un momento de su biografía por esta enfermedad (1,10-13, 18). Por tanto, hemos asistido a una evolución muy importante. En el resto de la Unión Europea, la evolución ha sido muy similar. Así, se ha pasado de adop-tar medidas disciplinarias que podían llegar al despido (Grecia, Italia, Reino Unido o República de Irlanda), a la realización programas preventivos y asistenciales de muy diversa índole $(1,2)$.

Tomando como referencia a España, hemos de expresar que es quizás de las más avanzadas en el campo de las drogodependencias, muy posiblemente por la pronta implicación de las centrales sindicales $(11,18,19)$. A pesar de ello, a nuestro modo de ver, existen dos cuestiones especialmente controvertidas sobre este tema que no se encuentran resueltas del todo. Por un lado, tendríamos la obligación moral de desarrollar por parte del empleador programas específicos de atención al trabajador toxicómano en la empresa que deberían ser promovidos por las diferentes Administraciones Autonómicas y, por otro, las campañas estructuradas sobre la determinación de drogas y alcohol dentro de la empresa, que a nuestro juicio sólo deberían efectuarse cuando las características del trabajo lo requirieran (10).

Siendo un objetivo común de los ordenamientos jurídicos la protección y la promoción de la salud de nuestros ciudadanos, es lógico que los Estados miembros de la Unión Europea vayan abordando y desarrollando paulatinamente diferentes respuestas a los problemas específicos del abuso del alcohol y de las drogas en el lugar de trabajo. En España, estos derechos a la protección y promoción de la salud se encuentran recogidos en la Constitución, Estatuto de los Trabajadores, Ley de Prevención de Riesgos Labo- rales, Ley General de la Seguridad Social y Ley General de Sanidad.

En este contexto, toda empresa, por pequeña que sea, debe ofrecer a su trabajador con problemas de consumo de alcohol y/o otras drogas asesoramiento, respuesta elemental y mínima que la empresa debe y puede asumir. Cuando las características de la empresa, su magnitud o su grado de implicación así lo decidan podrán además desarrollar actividades de educación y, o, tratamiento, interno o externo $(1,10)$.

El otro gran tema no lo suficientemente claro, se refiere a la determinación de alcohol y drogas en la empresa. Hemos de tener en cuenta que sólo se deberán hacer estas determinaciones cuando se trate de trabajos de riesgo, cualquiera que sea la forma acordada con la representación sindical, ya sea en los reconocimientos de ingreso o periódicos, o mediante muestreos aleatorios o sistemáticos (10). Sin embargo, las finalidades de la práctica de estas pruebas debe ser la de utilizar un método disuasorio que evite el consumo de alcohol y drogas, tanto en el trabajo como en las horas previas al comienzo del mismo y, por ende, garantizar la seguridad de aquellos trabajos de riesgo importante $(4,10,14)$. Nunca con la finalidad de un despido en caso de positividad y si la de la incorporación a un programa de tratamiento. Aquí subyace el miedo y las reservas a veces justificadas por parte de los representantes de los trabajadores (18, 19). Hemos de dar por supuesto, que además la empresa o quienes prestan la vigilancia de la salud, ya sea una mutua o un servicio de prevención ajeno, realizan otros programas preventivo-asistenciales.

A pesar de que algunos países sitúan la protección de los derechos individuales dentro de su ordenamiento jurídico en un rango de prioridad muy elevado, de forma que sólo está tolerada la realización de pruebas de detección ante trabajos de alto riesgo que puedan verse afectados por el consumo de alcohol y drogas (Bélgica, Francia, España, Grecia, Luxemburgo, Reino Unido), existen algunos países que han permitido la realización de pruebas de detección para el ingreso a puestos como cajero de banco o profesores (Italia) (2).

En este sentido, el sector económico de mayor siniestralidad en España es el de la Construcción, con una alta tasa de mortalidad. Existe la evidencia que los accidentes en la construcción suelen presentar una mayor incidencia en las horas posteriores a los descansos (bocadillo, comida). En España, se suscitó en 1999 una polémica en torno a la realización de la determinación de alcohol a estos trabajadores, que parece estar apaciguada en la actualidad. Nos parece 
correcta, si se encuentra dentro de un programa preventivo, con formación adecuada, y se les ofrece los recursos asistenciales apropiados; y no se nos escapa, la característica de temporalidad de este tipo de trabajos, con el consiguiente riesgo de sufrir un rechazo ante nuevas contrataciones para futuras obras. En nuestro espacio cultural, sólo Dinamarca parece haber encarado este problema con decisión (2).

Es importante precisar que algunas de estas pruebas se realizan, no con métodos analíticos en los medios biológicos, sino con métodos de detección basados en indicadores laborales, como la valoración del rendimiento, las alteraciones comportamentales, etc. (Bélgica, Dinamarca, Francia, Italia) $(1,2)$.

Por otro lado, hemos de tener presente que cualquier actuación en esta materia, en la empresa, como en otros problemas, tiene que estar consensuada y pactada con la representación sindical. Lo ideal sería reflejarlo en el Convenio Colectivo o en el Reglamento de Régimen Interior, mediante normativas que difundidas adecuadamente entre los trabajadores, facilitarán la adopción de criterios uniformes a la hora de enfocar estos temas $(1,2,10,11,18,19)$. Una actuación en este sentido, sin esta premisa no sólo estará condenada al fracaso con toda probabilidad, sino que lo más seguro es que sea motivo de un grave conflicto colectivo. Además, hemos de dejar claro que para todas estas actuaciones, damos por supuesto unos mínimos éticos de confidencialidad, voluntariedad, conocimiento, etc. cuya cita nos parece oportuna pero no su enumeración por sobrepasar la finalidad de este artículo $(10,12,20,21)$.

La rápida evolución social unida a los cambios en los patrones de conducta, en las actitudes sociales y en la conceptualización de los problemas han determinado un cambio continuo en las respuestas de nuestra sociedad a los problemas de las toxicomanías y del abuso de sustancias. La salud laboral y la medicina del trabajo, no han permanecido ajenas a esas tensiones y a la evolución antes descrita. Las interrogantes se suscitan en la eficacia real de nuestras soluciones y en la capacidad de evolucionar y de adaptarnos a las condiciones objetivas de una realidad dinámica. Es evidente que un problema complejo requiere soluciones al menos diversificadas y adaptadas a las peculiaridades del mismo, hablamos de problemas de salud en sentido amplio, que abarcan no solo la intervención médica restringida al diagnostico y, o, tratamiento, sino a una estrategia preventiva que incluye la incorporación y movilización de todos los participantes e integrantes del mundo laboral. Convertir a los trabajadores en agentes activos que participan en la búsque- da y diseño de las soluciones, es algo mas que un deseo ingenuo, o una frase retórica, eso debería ser el camino de incorporación de recursos y soluciones absolutamente necesarios. De otra parte el trabajo es uno de los recursos terapéuticos que adecuadamente dosificados, nos sirve para mantener en muchos casos un punto de anclaje en situaciones de riesgo de ruptura de una inserción sociofamiliar precaria. Todo esto nos obliga a incorporar e implicar en las actuaciones de prevención y de detección a otros agentes no sanitarios, pertenecientes al mundo laboral (trabajadores, representantes sindicales, etc.). En este sentido, hemos de expresar que, después del servicio médico, las principales vías de detección de trabajadores con problemas de consumo de alcohol y, o, drogas en la empresa Red Nacional de los Ferrocarriles Españoles son los trabajadores: los mandos, los representantes sindicales y otros compañeros (11).

Estamos convencidos de que una respuesta integrada y coordinada es una respuesta eficaz, y necesitamos de estrategias eficientes que nos proporcionen soluciones que serán tanto mas completas, cuanto mayor sea el grado de implicación de todos los agentes. Necesitamos transmitir la esperanza y la certeza de que la aplicación de medidas coherentes quizás no sea la solución total, pero si el mejor camino para lograrla (13).

La existencia de diversos estudios a nivel Nacional sobre la prevalencia del consumo de drogas en el medio laboral refieren algo menos de un 50\% de trabajadores que fuman, con una escalada del consumo por parte de la mujer, de un $15 \%$ de consumidores excesivos de alcohol, que también presumimos que puede encontrarse la mujer trabajadora en una proporción importante, de un $10 \%$ de consumidores de medicamentos sin prescripción médica específica, y de un 3-4\% de consumidores de las drogas no institucionalizadas (cannabis, cocaína, anfetaminas, éxtasis, heroína y sus mezclas) $(19,22)$. Datos que es posible encontrar en cualquier estudio que realicemos en nues-tro propio medio (tabla 1) $(5,6)$. Estos resultados justifican el desarrollo de Leyes por parte de las Administraciones y la realización de actividades preventivas por parte de las empresas, Asociaciones Empresariales y Centrales Sindicales con la finalidad de continuar en la sensibilización de todos los sectores implicados en identificar el trabajador con problemas de consumo y desviarlo a Centros Asistenciales especializados para su tratamiento, recuperarlo psíquica y físicamente y reinsertarlo social y laboralmente $(1,2,10,13,19$ 21). 
TABLA 1. Evolución de la enfermedad alcohólica y de las otras toxicomanías observadas por nosotros en nuestra empresa siguiendo diferentes criterios.

\begin{tabular}{|c|c|c|c|c|c|c|}
\hline PERIODO & DROGAS & ALCOHOL & TABACO & ESTUDIO & PERSONAL & OBJETIVO \\
\hline $1988-92$ & $3.6 \%$ & $8.4 \%$ & & $\mathrm{~N}=800$ & $\mathrm{~N}=1.357$ & Prevalencia \\
\hline $1988-92$ & & $8.6 \%$ & $51.3 \%$ & $\mathrm{~N}=152$ & $\mathrm{~N}=405$ & Test de Le Gô \\
\hline $1993-95$ & $1.4 \%$ & $5.6 \%$ & & $\mathrm{~N}=993$ & $\mathrm{~N}=1.208$ & $\begin{array}{c}\text { Reinserción } \\
\text { profesional }\end{array}$ \\
\hline $1996-97$ & $1.1 \%$ & $11.4 \%$ & $50.6 \%$ & $\mathrm{~N}=650$ & $\mathrm{~N}=1.063$ & $\begin{array}{c}\text { Consumo etanol }> \\
280 \text { mL/semana }\end{array}$ \\
\hline 1999 & $0.0 \%$ & $9.9 \%$ & $50.0 \%$ & $\mathrm{~N}=193$ & $\mathrm{~N}=913$ & $\begin{array}{c}\text { Evaluar encuesta de } \\
\text { consumo/CAGE }\end{array}$ \\
\hline
\end{tabular}

\section{BIBLIOGRAFIA:}

1. Organización Internacional del Trabajo. Alcohol y drogas. Programas de asistencia a los trabajadores (informes). Madrid: AGISA, 1989.

2. Daintith T, Baldwin R. Alcohol y drogas en el lugar de trabajo. Análisis de la legislación y normativa de los Estados miembros de la Comunidad Europea. Institute of Avanced Legal Studies, 1993.

3. Ley 31/1995, de 8 de noviembre, de Prevención de Riesgos Laborales.

4. Cabrero E, Osuna E, Luna JD, Jiménez-Bueno MC, Box M, Luna A. Estudio de un muestreo de alcohol exhalado en aire alveolar en una pobla-ción de la Red Nacional de los Ferrocarriles Espa oles. Adicciones, 1994; 6: 1522.

5. Cabrero E, Barrios I, Osuna E, Luna JD, Jiménez-Bueno MC, Luna A. Detección del bebedor excesivo en el medio laboral mediante el test de Le Gô. Adicciones, 1996; 8: 321-329.

6. Cabrero E, Osuna E, Luna JD, Jiménez-Bueno MC, Blanco A, Luna A. Consecuencias de las drogodependencias en el mundo laboral. Adicciones, 1996; 8: 419-428.
7. Cabrero E, Osuna E, Luna JD, Jiménez MC, Blanco A, Luna A. Alcoolis-me, absentéisme et accidents du travail dans une entreprise de transport espagnole. Arch mal prof, 1996; 57: 353-355.

8. Gea C, Pérez-Cárceles MD, Osuna E, Cabrero E, Luna A. Prevalencia del abuso de alcohol en una población laboral del sector pesquero. Adicciones, 1996; 8: 267276.

9. Gómez-Acebo A. Drogas y Trabajo: Actuación de los Servicios Médicos de Empresa. Problemática actual de los Seurvicios Médicos de Empresa ante el consumo de drogas y trabajo. Med Segur Trab, 1987; 34: 16-20.

10. Cabrero E, Luna A. Determinación de alcohol y drogas en la empresa, Rev Esp Drogodep, 1999; 24: 3-16.

11. Cabrero E. Estamentos laborales y detección, tratamiento y reinserción de drogodependientes en el medio laboral, Adicciones, 1999, 11 (Suplemento 1): 8.

12. Cabrero E, Luna A. Ética y Medicina del Trabajo, Medicina del Trabajo, 1999, 8: 78-83.

13. Cabrero E, Gómez-Acebo A, García-Alcázar I, GarcíaAlbacar A, Luna JD, Luna A. Programme contre la toxicomanie et I'alcoolisme dans l'entreprise ferroviaire espagnole <RENFE>, 1990-97", Arch Mal Prof, 2000; 61: 310-317. 
14. Cabrero E, Gómez-Acebo A, García-Alcázar I, GarcíaEsquinas N, Luna JD, Luna A. Muestreos sistemáticos de alcohol en aire expirado en una empresa de transporte ferroviario, Med Segur Trab, 1998; 179: 23-30.

15. Trarieux R, Coulondre D. Toxicomanies, problèmes de dépistage en milieu de travail et implications dans l'aptitude. Arch mal prof, 1995; 56: 564-566.

16. Wang CCK, Poe HK. Pruebas del alcohol y drogas en los empleados como función de la gestión de la seguridad. Notas y documentos del Instituto Nacional de Medicina y Seguridad en el Trabajo (número 1144), 1993; 3: 7179.

17. Real Decreto Legislativo 1/1995, de 24 de marzo, por el que se aprueba el texto refundido de la Ley del Estatuto de los Trabajadores, Ley 8/1980, de 8 de marzo.
18. Comisiones Obreras. Orientaciones sobre intervención sindical en drogodependencias. Madrid: Secretaría Confederal de Acción Sindical, 1992.

19. Unión General de Trabajadores, Equipo de Investigaciones Sociológicas. Drogodependencias. Madrid: Unión General de Trabajadores, 1987.

20. American Occupational Medical Association (Committee Report). Drugs Screening in the Workplace: Ethical Guidelines. J Occup Med, 1986; 28: 1240-1241.

21. American College of Occupational Medicine (Council of Social Issues). Drugs Screening in the Workplace: Ethical Guideli-nes. J Occup Med, 1991; 33: 651-652.

22. Fundación de Ayuda contra la Drogadicción, Equipo de Investigaciones Sociológicas. La incidencia de las drogas en el mundo laboral, 1996. Madrid: Fundación de Ayuda contra la Drogadicción, 1996. 